\title{
Role of the $h_{1}(1800)$ and $f_{1}(1285)$ states in the $J / \psi$ decays
}

\section{Ju-Jun Xie*}

Institute of modern physics, Chinese academy of sciences

E-mail: xiejujun@impcas.ac.cn

\section{Miguel Albaladejo}

Departamento de Física, Universidad de Murcia, E-30071 Murcia, Spain

\section{Eulogio Oset}

Departamento de Física Teórica and IFIC, Centro Mixto Universidad de Valencia-CSIC Institutos de Investigación de Paterna, Aptdo. 22085, 46071 Valencia, Spain

The BES data on the $J / \psi \rightarrow \eta K^{* 0} \bar{K}^{* 0}$ reaction show a clear enhancement in the $K^{* 0} \bar{K}^{* 0}$ mass distribution close to the threshold of this channel. Such an enhancement is usually a signature of a resonance around threshold, which in this case would correspond to an $h_{1}$ state with quantum numbers $I^{G}\left(J^{P C}\right)=0^{-}\left(1^{+-}\right)$. This state around $1800 \mathrm{MeV}$ is studied from the interaction of the $K^{* 0} \bar{K}^{* 0}$ in $J / \psi \rightarrow \eta K^{* 0} \bar{K}^{* 0}$ decay, confirming the relationship of the enhancement in the invariant mass spectrum with the $h_{1}$ resonance. The role of $f_{1}(1285)$ resonance in the $J / \psi \rightarrow \phi \bar{K} K^{*}$ and $J / \psi \rightarrow \phi f_{1}(1285)$ decays are also investigated. The theoretical approach is based on the results of chiral unitary theory where the $f_{1}(1285)$ resonance is dynamically generated from the $\bar{K} K^{*}$ interaction. In order to further test the dynamical nature of the $f_{1}(1285)$ state, we investigate the $J / \psi \rightarrow \phi \bar{K} K^{*}$ decay close to the $\bar{K} K^{*}$ threshold and make predictions for the ratio of the invariant mass distributions of the $J / \psi \rightarrow \phi \bar{K} K^{*}$ decay and the $J / \psi \rightarrow \phi f_{1}(1285)$ partial decay width with all the parameters of the mechanism fixed in previous studies. The results can be tested in future experiments and therefore offer new clues on the nature of the $h_{1}(1800)$ and $f_{1}(1285)$ states.

XVII International Conference on Hadron Spectroscopy and Structure - Hadron2017

25-29 September, 2017

University of Salamanca, Salamanca, Spain

\footnotetext{
${ }^{*}$ Speaker.
} 


\section{Introduction}

Since the $J / \psi$ and the $\eta$ mesons have quantum numbers $I^{G}\left(J^{P C}\right)=0^{-}\left(1^{--}\right)$and $0^{+}\left(0^{-+}\right)$, respectively, the decay $J / \psi \rightarrow \eta K^{* 0} \bar{K}^{* 0}$ constitutes the ideal reaction to look for an $h_{1}$ state, with quantum numbers $0^{-}\left(1^{+-}\right)$. However, both on the theoretical and experimental sides, the study of $h_{1}$ states above $1.5 \mathrm{GeV}$ is very scarce [1]. In Refs. [2, 3], the vector-vector interaction generates resonances in different strangeness-isospin-spin channels. In the $0^{-}\left(1^{+-}\right)$sector, a resonance was found with mass and width around 1800 and $80 \mathrm{MeV}$, respectively, which is dynamically generated from the interaction of $K^{*}$ and $\bar{K}^{*}$. This state cannot couple to other vector-vector or pseudoscalarpseudoscalar channels, which makes its observation difficult. Furthermore, this state cannot be clearly identified with any of the $h_{1}$ states listed in the PDG [1], and it is not found in Ref. [4]. A different dynamical approach to the vector-vector interactions is provided in [5], using a massive Yang-Mills field theoretical framework. On the experimental side, the BES results [6] shows an enhancement in the invariant mass spectrum of the $K^{* 0} \bar{K}^{* 0}$ around $1850 \mathrm{MeV}$, which might hint to a sizeable contribution from an $h_{1}$ state strongly coupling to the $K^{* 0} \bar{K}^{* 0}$ channel.

The $f_{1}(1285)$ resonance with quantum numbers $0^{+}\left(1^{++}\right)$is an axial-vector state, which is described as a $q \bar{q}$ state $[7,8,9,10,11,12]$. On the other hand, the $f_{1}(1285)$ is also suggested to be a dynamically generated state from the single channel $\bar{K} K^{*}$ interaction in the chiral unitary approach [13]. Because $f_{1}(1285)$ resonance has positive $G$ parity, it cannot couple to other pseudoscalar-vector channels and two pseudoscalar mesons. Thus, since $f_{1}(1285)$ is located below the $\bar{K} K^{*}$ mass threshold, its observation is difficult in two body decays. Indeed, the main decay channels of the $f_{1}(1285)$ are $4 \pi$ (branching ratio $\left.=33 \%\right), \eta \pi \pi(52 \%)$, and $\pi \bar{K} K(9 \%)$. In Refs. [14, 15], the decays of $f_{1}(1285) \rightarrow \eta \pi^{0} \pi^{0}$ and $f_{1}(1285) \rightarrow \pi K \bar{K}$ were studied using the $\bar{K} K^{*}$ picture of the $f_{1}(1285)$. The theoretical predictions are compatible with the experimental measurements. Recently, the production of $f_{1}(1285)$ resonance in the reaction $K^{-} p \rightarrow f_{1}(1285) \Lambda$ within an effective Lagrangian approach was studied in Ref. [16] based on the results obtained in chiral unitary theory. The theoretical calculations are also in agreement with the experimental data.

On the experimental side, in Refs. [17, 18], the decay of $J / \psi \rightarrow \phi f_{1}(1285)$ was studied from the $J / \psi \rightarrow \phi 2\left(\pi^{+} \pi^{-}\right)$and $J / \psi \rightarrow \phi \eta \pi^{+} \pi^{-}$decays by the DM2 Collaboration, while in Ref. [19], the branching fraction of $J / \psi \rightarrow \phi \bar{K} K^{*}$ was measured from the decay of $J / \psi \rightarrow \phi K \bar{K} \pi$ by the BES Collaboration. Because the $J / \psi$ and the $\phi$ mesons have quantum numbers $0^{-}\left(1^{--}\right)$and $0^{-}\left(1^{--}\right)$, respectively, the decay $J / \psi \rightarrow \phi \bar{K} K^{*}$ constitutes the ideal reaction to look for the $f_{1}(1285)$ state, with quantum numbers $0^{+}\left(1^{++}\right)$, coupling to an $s$ wave $\bar{K} K^{*}$ pair. However, since the $f_{1}(1285)$ is located below the $\bar{K} K^{*}$ threshold, it will contribute to the region close to the threshold of $\bar{K} K^{*}$.

\section{The decay of $J / \psi \rightarrow \eta K^{* 0} \bar{K}^{* 0}$}

The decay mechanism is shown in Fig. 1. We assume that, the bare production vertex for $J / \psi \rightarrow \eta K^{* 0} \bar{K}^{* 0}$, is of a short range nature, i.e., just a coupling constant in the field theory language. Then we can easily get the $K^{* 0} \bar{K}^{* 0}$ invariant mass spectrum for the $J / \psi \rightarrow \eta K^{* 0} \bar{K}^{* 0}$ as [20]:

$$
\frac{d \Gamma}{d M_{\mathrm{inv}}}=\frac{C}{v\left(M_{\mathrm{inv}}^{2}\right)} \frac{p_{1} \widetilde{p}_{2}}{M_{J / \psi}} t\left(M_{\mathrm{inv}}^{2}\right)
$$




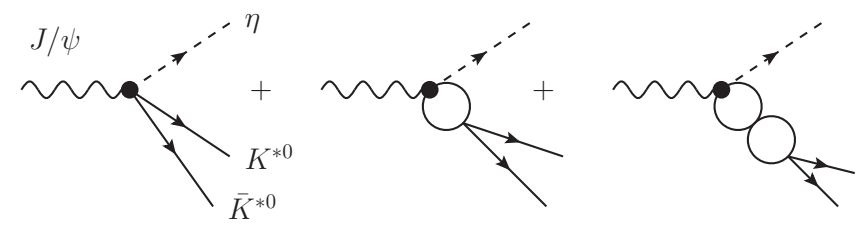

Figure 1: Diagrammatic representation of the $J / \psi \rightarrow \eta K^{* 0} \bar{K}^{* 0}$ decay.

with $C$ a global constant factor which will be fitted to the data. The $t$ and $v$ stand for the $K^{*} \bar{K}^{*} \rightarrow$ $K^{*} \bar{K}^{*}$ scattering amplitude and transition potential, respectively, which depend on the invariant mass, $M_{\text {inv }}$ of $K^{*} \bar{K}^{*}$ system. And $p_{1}$ is the $\eta$ momentum in the $J / \psi$ rest frame, while $\widetilde{p}_{2}$ is the convolution with the mass distribution of the two $K^{* 0}$, of the momentum of $K^{* 0}$ in the center mass frame of $K^{* 0}$ and $\bar{K}^{* 0}$ system (see more details in Ref. [21]).

There are two free parameters for calculating the $K^{* 0} \bar{K}^{* 0}$ invariant mass spectrum. In model $\mathrm{A}$, one is the global constant $C$. The other one is the subtraction constant $a(\mu)$, which determines the amplitude $t$. We find a good agreement with the data [6] using the natural range $-1.0<$ $a(\mu)<-0.6$. In addition, we consider a constant potential $v$ (model $\mathrm{B}$ ), fitted to reproduce the data. A constant potential is acceptable here given the small range of $M_{\text {inv }}$ considered in this work, and would render our analysis more model independent. By considering a constant potential, the subtraction constant $a(\mu)$ is no longer a free parameter, and we fix $a(\mu)=-0.8$ in this case. Then, we have two free parameters need to be determined: the global constant $C$, and the potential $v$ itself.

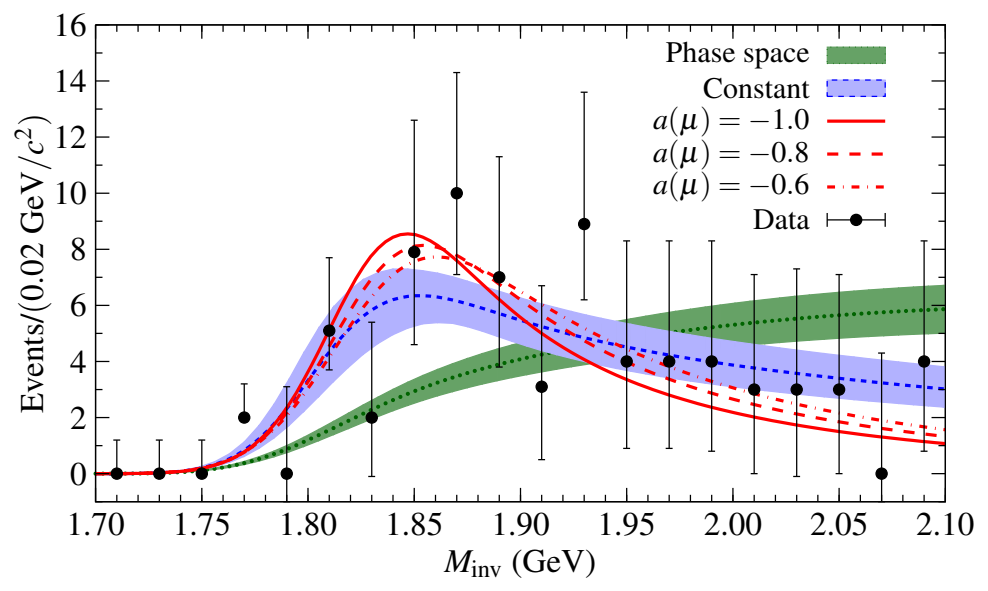

Figure 2: The $K^{* 0} \bar{K}^{* 0}$ invariant mass spectrum of $J / \psi \rightarrow \eta K^{* 0} \bar{K}^{* 0}$ decay.

In both approaches, the free parameters are fitted by means of a $\chi^{2}$ function to reproduce the $K^{* 0} \bar{K}^{* 0}$ invariant mass spectrum data of the BES Collaboration, shown in Fig. 2, where the blue short-dashed curve represents the spectrum obtained with the fitted parameters of model B. One can see that the reproduction of the data is good. We stress that this approach is rather model independent, since we do not assume any underlying model for the transition potential $v$, but take it to be a constant. For model A, the results are shown in Fig. 2 with solid, long-dashed, and dotdashed lines, with three different values of the subtraction constant, $a(\mu)=-1.0,-0.8$ and -0.6 , respectively. It is also clear that the reproduction of the data is also good. Finally, the (green) 
dotted line, and the associated error band (dark green) is the prediction for phase space alone. It is find that the inclusion of an $h_{1}$ state, generated dynamically from the $K^{*} \bar{K}^{*}$ interaction, is crucial to achieve a fairly good description of the BES data. The phase space alone clearly does not follow the trend of the data. But one observes that below $1.93 \mathrm{GeV}$ almost every datum is above the phase space band, while they are mostly below for energies above that one.

\section{The decay of $J / \psi \rightarrow \phi \bar{K} K^{*}$}

For $J / \psi \rightarrow \phi \bar{K} K^{*}$, the decay mechanism is similar as the case of $J / \psi \rightarrow \eta K^{* 0} \bar{K}^{* 0}$ as shown in Fig. 1, replacing $\eta$ with $\phi$ and $\bar{K}^{* 0}$ with $\bar{K}$. With the scattering amplitude $t$ and the transition potential $v$ for $\bar{K} K^{*} \rightarrow \bar{K} K^{*}$, we depend on the invariant mass, $M_{\text {inv }}$, of $\bar{K}$ and $K^{*}$, the decay amplitude for $J / \psi \rightarrow \phi \bar{K} K^{*}$ is given by

$$
T_{J / \psi \rightarrow \phi \bar{K} K^{*}}=V_{P} C_{s}\left[1+G\left(M_{\mathrm{inv}}^{2}\right) t\left(M_{\mathrm{inv}}^{2}\right)\right]=V_{P} C_{s} \frac{t\left(M_{\mathrm{inv}}\right)}{v\left(M_{\mathrm{inv}}\right)},
$$

with $V_{P}$ a constant and $C_{s}$ is the spin structure: $C_{s}=\varepsilon_{i j k} \varepsilon_{i}(J / \psi) \varepsilon_{j}(\phi) \varepsilon_{k}\left(K^{*}\right)$. Then, we can easily get the $\bar{K} K^{*}$ invariant mass spectrum for the $J / \psi \rightarrow \phi \bar{K} K^{*}$ as [20, 21, 22]:

$$
\frac{d \Gamma_{J / \psi \rightarrow \phi \bar{K} K^{*}}}{d M_{\mathrm{inv}}}=\frac{V_{p}^{2}}{(2 \pi)^{3}} \frac{M_{\mathrm{inv}}}{8 M_{J / \psi}^{3}} \frac{t\left(M_{\mathrm{inv}}\right)}{v\left(M_{\mathrm{inv}}\right)} \int_{M_{\phi \bar{K}}^{\min }}^{M_{\phi \bar{x}}^{\max }} \bar{\sum} \sum C_{s}^{2} M_{\phi \bar{K}} d M_{\phi \bar{K}} .
$$

For a given value of $M_{\text {inv }}$, the range of $M_{\phi \bar{K}}$ can be easily obtained as explicitly shown in Ref. [23].

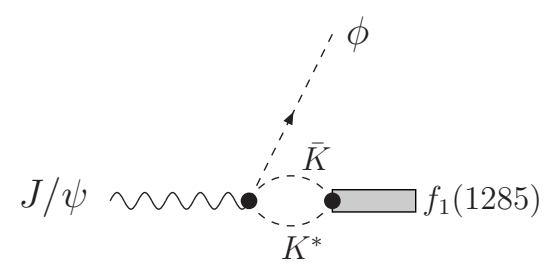

Figure 3: Production mechanism of the $J / \psi \rightarrow \phi f_{1}(1285)$ decay.

If we are interested in the production of the $f_{1}(1285)$ resonance, the relevant mechanism is depicted diagrammatically in Fig. 3. Then, the partial decay width of $J / \psi \rightarrow \phi f_{1}(1285)$ is

$$
\Gamma_{J / \psi \rightarrow \phi f_{1}(1285)}=\frac{V_{P}^{2}}{8 \pi} \frac{G^{2}\left(M_{f_{1}}\right) g_{f_{1}}^{2} p_{\phi}^{\prime}}{M_{J / \psi}^{2}} \sum \sum C_{s}^{\prime 2} .
$$

where the spin factor $C_{s}^{\prime}$ is now $C_{s}^{\prime}=\varepsilon_{i j k} \varepsilon_{i}(J / \psi) \varepsilon_{j}(\phi) \varepsilon_{k}\left(f_{1}\right)$. The $g_{f_{1}}=7555 \mathrm{MeV}$ is the coupling constant of the $f_{1}(1285)$ to the $\bar{K} K^{*}$ channel.

The chiral theory cannot provide the value of the constant $V_{P}$ in Eqs. 3.2 and 3.3, however, if we divide $d \Gamma / d M_{\text {inv }}$ by $\Gamma_{J / \psi \rightarrow \phi f_{1}(1285)}$ the constant $V_{P}$ is cancelled, and we can make precise predictions for the ratio $R_{\Gamma}=\frac{d \Gamma_{J / \psi \rightarrow \phi \bar{K} K^{*}} / d M_{\text {inv }}}{\Gamma_{J / \psi \rightarrow \phi f_{1}(1285)}}$. This ratio is relevant because it has no free parameters (all the parameters are fixed by previous works) and, thus, it is a prediction of the theory. The shape, as well as the absolute values of the ratio $R_{\Gamma}$ for the $\bar{K} K^{*}$ mass distribution, can be compared with the experimental measurements. 


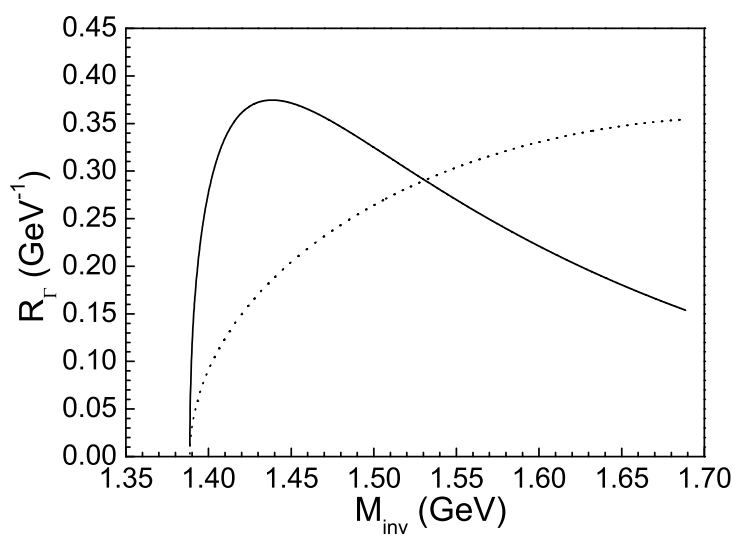

Figure 4: Results of $R_{\Gamma}$ as a function of invariant mass $M_{\text {inv }}$ of $\bar{K} K^{*}$.

In Fig. 4, we show the theoretical results for $R_{\Gamma}$ as a function of $M_{\text {inv }}$. The solid curve stands for the theory prediction and the dotted curve stands for the phase space. For evaluating the contributions of the phase space, we replace $t\left(M_{\mathrm{inv}}\right) / v\left(M_{\mathrm{inv}}\right)$ of Eq. 3.1 by a constant, thus removing any effect of the $M_{\text {inv }}$ dependence of the $f_{1}(1285)$ resonance. Then we tune this constant such that the $M_{\text {inv }}$ integrated $R_{\Gamma}$ in the range of energies from the $\bar{K} K^{*}$ threshold to $1.7 \mathrm{GeV}$ is the same as the one evaluated with the explicit resonance formalism. We see a clear threshold enhancement in Fig. 4 which is caused by the contributions of the $f_{1}(1285)$ state below threshold, which is dynamically generated by the $\bar{K} K^{*}$ interaction. Actually, the range of the invariant mass of $\bar{K} K^{*}$ in the decay of $J / \psi \rightarrow \phi \bar{K} K^{*}$ is from the threshold of $\bar{K} K^{*}$ up to $2.077 \mathrm{GeV}$, however, we cannot go so far because the chiral theory works well about $200-300 \mathrm{MeV}$ from the threshold, hence we consider only the range of $300 \mathrm{MeV}$ above the $\bar{K} K^{*}$ threshold as shown in Fig. 4. The future experimental observation of the mass distribution $R_{\Gamma}$ would provide very valuable information on the mechanism of the $J / \psi \rightarrow \phi \bar{K} K^{*}$ decay.

\section{Summary}

In summary, we have studied the $J / \psi \rightarrow \eta K^{* 0} \bar{K}^{* 0}, \rightarrow \phi \bar{K} K^{*}$ and $\rightarrow \phi f_{1}(1285)$ with the aim of determining the existence of an $h_{1}$ state and the nature of $f_{1}(1285)$. By using a constant potential and a local hidden gauge potential for the $K^{*} \bar{K}^{*} \rightarrow K^{*} \bar{K}^{*}$ transition, we calculate the $K^{* 0} \bar{K}^{* 0}$ invariant mass spectrum. By fitting our results to the BES experimental data, we find that these can be fairly described by including the contributions from an $h_{1}$ state. The mass and width of this $h_{1}$ state are around $M_{h_{1}}=1830 \pm 20 \mathrm{MeV}$ and $\Gamma_{h_{1}}=110 \pm 10 \mathrm{MeV}$, respectively.

For the $J / \psi \rightarrow \phi \bar{K} K^{*}$ and $J / \psi \rightarrow \phi f_{1}(1285)$ decays, we calculate the ratio $R_{\Gamma}$ as a function of invariant mass $M_{\text {inv }}$ of $\bar{K} K^{*}$. A clear threshold enhancement in Fig. 4 compared with the phase space appears, which is caused by the presence of the $f_{1}(1285)$ state below threshold. The experimental observation of this mass distribution would then provide very valuable information to check our predictions and the basic nature of the $f_{1}(1285)$ resonance. 


\section{Acknowledgments}

This work is partly supported by the National Natural Science Foundation of China (Grants No. 11475227 and 11735003), the Youth Innovation Promotion Association CAS (No. 2016367), and the Spanish Ministerio de Economia y Competitividad and European FEDER funds under the contract number FIS2011-28853-C02-01, FIS2011- 28853-C02-02, FIS2014-57026-REDT, FIS2014-51948-C2- 1-P, and FIS2014-51948-C2-2-P, and the Generalitat Valenciana in the program Prometeo II-2014/068. We acknowledge the support of the European Community-Research Infrastructure Integrating Activity Study of Strongly Interacting Matter (acronym HadronPhysics3, Grant Agreement n. 283286) under the Seventh Framework Programme of EU.

\section{References}

[1] C. Patrignani et al. [Particle Data Group], Chin. Phys. C 40, 100001 (2016).

[2] L.S. Geng, and E. Oset, Phys. Rev. D 79, 074009 (2009).

[3] R. Molina, D. Nicmorus and E. Oset, Phys. Rev. D 78, 114018 (2008).

[4] C. Garcia-Recio, L. S. Geng, J. Nieves and L. L. Salcedo, Phys. Rev. D 83, 016007 (2011).

[5] M. Albaladejo and J. A. Oller, Phys. Rev. Lett. 101, 252002 (2008).

[6] M.Ablikim et al. [BES Collaboration], Phys. Lett. B 685, 27 (2010).

[7] P. Gavillet, R. Armenteros, M. Aguilar-Benitez, M. Mazzucato and C. Dionisi, Z. Phys. C 16, 119 (1982).

[8] S. Godfrey and J. Napolitano, Rev. Mod. Phys. 71, 1411 (1999).

[9] D. M. Li, H. Yu and Q. X. Shen, Chin. Phys. Lett. 17, 558 (2000).

[10] J. Vijande, F. Fernandez and A. Valcarce, J. Phys. G 31, 481 (2005).

[11] E. Klempt and A. Zaitsev, Phys. Rept. 454, 1 (2007).

[12] K. Chen, C. Q. Pang, X. Liu and T. Matsuki, Phys. Rev. D 91, 074025 (2015).

[13] L. Roca, E. Oset and J. Singh, Phys. Rev. D 72, 014002 (2005).

[14] F. Aceti, J. J. Xie and E. Oset, Phys. Lett. B 750, 609 (2015).

[15] F. Aceti, J. M. Dias and E. Oset, Eur. Phys. J. A 51, 48 (2015).

[16] J. J. Xie, Phys. Rev. C 92, 065203 (2015).

[17] A. Falvard et al. [DM2 Collaboration], Phys. Rev. D 38, 2706 (1988).

[18] J. Jousset et al. [DM2 Collaboration], Phys. Rev. D 41, 1389 (1990).

[19] M. Ablikim et al. [BES Collaboration], Phys. Rev. D 77, 032005 (2008).

[20] J. C. Nacher, E. Oset, H. Toki and A. Ramos, Phys. Lett. B 455, 55 (1999).

[21] J. J. Xie, M. Albaladejo and E. Oset, Phys. Lett. B 728, 319 (2014).

[22] A. Martinez Torres, K. P. Khemchandani, F. S. Navarra, M. Nielsen and E. Oset, Phys. Lett. B 719, 388 (2013).

[23] J. J. Xie and E. Oset, Phys. Lett. B 753, 591 (2016). 